\title{
Using Topiramate in Patients with Epilepsy: Practical Aspects
}

\author{
J.W.A.S. Sander
}

\begin{abstract}
Clinical trials are important in determining the relative efficacy and safety of a new antiepileptic drug (AED); however, experience acquired in clinical practice will eventually determine its position in the antiepileptic armamentarium. Topiramate (TPM), a new AED has been available in the United Kingdom since mid-1995 and a considerable number of patients have being treated. As a result of this experience, a number of changes have being made in the way TPM is used, particularly in the starting doses and titration rates. This seems to have improved patients' tolerability of treatment, an important consideration if a drug is to be used to its optimum level. In this article, practical tips for the use of TPM are given and these include starting doses, titration rates, options for managing side effects occurring early in treatment, advice concerning the withdrawal of concomitant AEDs and indications for discontinuation of TPM. The need for adequate patient counseling regarding potential side effects and expectations of treatment is also reviewed.
\end{abstract}

\begin{abstract}
RÉSUMÉ: Aspects pratiques de l'utilisation du topiramate chez les patients épileptiques. Les essais thérapeutiques sont importants pour déterminer l'efficacité relative et la sécurité d'un nouvel agent antiépileptique (AAE). Cependant, l'expérience acquise en pratique clinique déterminera éventuellement sa position dans la pharmacopée antiépileptique. Le topiramate (TPM), un nouveau AAE, est disponible au Royaume Uni depuis le milieu de 1995 et un nombre considérable de patients a reçu ce médicament. Suite à ces expériences, un certain nombre de changements ont été apportés à l'utilisation du TPM, particulièrement en ce qui concerne les doses initiales et la titration, ce qui semble avoir amélioré la tolérabilité du traitement, une considération importante si on veut utiliser le médicament de façon optimale. Dans cet article, nous fournissons des suggestions pratiques pour l'utilisation du TPM, incluant la posologie initiale, la titration, la façon de contrer les effets secondaires survenant tôt au cours du traitement, des conseils sur le sevrage d'AAEs concomitants et des indications pour l'arrêt du TPM. Nous discutons également de la nécessité de conseiller adéquatement les patients sur les effets secondaires et les bénéfices possibles du traitement.
\end{abstract}

Can. J. Neurol. Sci. 1998; 25: S16-S18

The eventual place of a new antiepileptic drug (AED) in the antiepileptic armamentarium evolves only gradually from controlled clinical trials through intense use in clinical practice. Clinical trials most reliably establish the relative efficacy and the general safety of an AED in a given target population and are essential for regulatory purposes. ${ }^{1}$ Outcome in such trials is usually measured in terms of a $50 \%$ reduction in seizure frequency. The highly selective entry criteria and strict procedures laid out in the protocols for such trials assure the homogeneity of treatment groups, study drug administration, duration of therapy and clinical and laboratory assessments required to perform valid between-group statistical comparisons of efficacy and safety measures. At the same time, the specificity and inflexibility of controlled trial protocols provide only limited information on the practical day-to-day use of the drug when complete seizure freedom with no side effects is the desirable outcome of treatment. $^{2}$

Whilst open clinical trial experience is inherently less reliable than controlled trial experience, unblinded studies nevertheless provide some opportunities to uncover valuable new information concerning the appropriate use of a new agent. Examples include the exploration of dosage ranges and dosing schedules not provided for in controlled trials and testing of alternative strategies for managing side effects, such as the reduction or elimination of concomitant AED medication. Thus, both controlled and open trials contribute significantly to the knowledge base required for optimal use of a newly available AED in clinical practice. The least scientific but arguably the most valuable opportunity to expand our knowledge concerning the practical use of any new treatment arises after the drug is approved and marketed. It can then be used in a real clinical scenario and for instance, offered to patients who decline participation in clinical trials or do not meet all of the entry requirements of formal protocols.

Topiramate (TPM) is a structurally novel AED initially approved in the United Kingdom in mid-1995. At that time, TPM had been administered to approximately 2000 patients in controlled and open trials, more than 100 of whom had been treated continuously with TPM for 5-7 years. In order to develop clinical guidelines concerning practical aspects of the use of

From the Epilepsy Research Group, Institute of Neurology, National Hospital for Neurology and Neurosurgery, Queen Square, London, United Kingdom.

Reprint requests to: J.W.A.S. Sander, Epilepsy Research Group, National Hospital for Neurology and Neurosurgery, 33 Queen Square, London WCIN 3B6, United Kingdom. 
TPM, we reviewed the available overall results of controlled and open studies of TPM completed to date. We have also considered our clinical experience with TPM in more than 500 patients at the National Hospital for Neurology and Neurosurgery (Queen Square and Chalfont sites) epilepsy clinics since its general availability in the United Kingdom, and formulated observations and guidelines relevant to the optimal use of TPM in clinical practice.

\section{Clinical efficacy}

Results of controlled clinical trials conducted to date have demonstrated the efficacy of TPM as adjunctive therapy in adults with partial seizures with or without secondary generalization. ${ }^{3-5}$ Initial experience also suggests that TPM can be used as AED monotherapy in partial epilepsy. ${ }^{7}$ Positive but anecdotal reports concerning the efficacy of TPM in aduits with generalized seizures ${ }^{7}$ and in children demonstrating various types of seizures ${ }^{8}$ have recently been confirmed in formal controlled clinical trials.

\section{Clinical safety}

The most common adverse events reported during clinical trials of TPM as add-on therapy and also seen in clinical practice have been central nervous system (CNS) symptoms. ${ }^{3.5}$ These include dizziness, somnolence, slowed mentation, nervousness, paresthesia, fatigue, impaired cognition and confusion. These are usually transient resolving within a week. Renal stones, most of which passed spontaneously, have occurred in approximately $1.5 \%$ of patients treated with TPM in clinical trials $^{9}$ but seem to be less common in clinical practice. Weight loss reported during TPM therapy is common affecting as many as a third of patients. It is usually mild, appears to be related to baseline weight, and is at least partially reversible in some patients after prolonged therapy. ${ }^{9}$

\section{Clinical relevance of titration schedule}

In placebo-controlled trials of TPM as add-on therapy in partial epilepsy, most of the adverse events observed with TPM tended to appear early in treatment and resolve with continuing therapy. ${ }^{3-5}$ The early appearance of these events appears to be related to the forced rapid titration schedules associated with these trials, wherein increases of 100-200 mg of TPM weekly were required by the study protocols. An analysis of study discontinuations among 360 patients who received TPM in controlled trials showed that approximately three-fourths of those who experienced limiting adverse events dropped out within the first 2 months of therapy, most frequently during the rapid dosage titration period. ${ }^{9}$ More recently, clinicians have had the opportunity to employ slower TPM titration schedules with rewarding results.

\section{Practical guidelines}

\section{Dosage of TPM}

Dosage guidelines developed for optimizing the utility of TPM are summarized in the Table. The recommended starting dosage of TPM as adjunctive therapy is $25 \mathrm{mg} /$ day after which titration can proceed in $25 \mathrm{mg} /$ day increments at two-weekly intervals up to $200 \mathrm{mg} /$ day (100 mg bid). Thereafter, the daily dosage may be increased by $50 \mathrm{mg}$ each week until clinical efficacy is achieved or the maximum tolerated dosage of TPM is reached. The minimum effective dosage of TPM as add-on therapy in refractory partial epilepsy is generally considered to be $200 \mathrm{mg} /$ day and the effective dosage range for most patients is $200-400 \mathrm{mg} /$ day. However, there are patients responding to as little as $50 \mathrm{mg} /$ day, while a few have received dosages of up to $1600 \mathrm{mg} /$ day of TPM.

\section{Side effect management}

A number of strategies are available for dealing with the occurrence of CNS adverse events encountered during TPM therapy, depending on their severity. If side effects occur during the titration period, the usual practice is to delay further dosage increases for 1-2 weeks or until symptoms are not longer present. Other options include reducing the dosage of TPM to the previous dose level or reducing the dosage of a concomitant AED. Titration of TPM may be resumed when the side effect resolves.

\section{Maintenance therapy}

Increases in the dosage of TPM may be considered when the patient is not seizure free but has demonstrated some reduction in seizure frequency or severity and is not experiencing limiting side effects. When the optimal dosage of TPM is reached, dose reduction or withdrawal of concomitant AEDs may be attempted. It should be noted that withdrawal of an enzyme-inducing AED such as carbamazepine (CBZ) or phenytoin (PHT) may result in up to a two-fold increase in the blood level of TPM, ${ }^{10}$ and re-adjusting the dose of TPM downwards should be considered if side effects are an issue. If seizure frequency increases or there is recurrence after discontinuation of concomitant AEDs, a low dose of the previous AED may be beneficial.

\section{Discontinuation of TPM}

Discontinuation of TPM should be considered if side effects continue after dose reduction or at any point become unacceptable to the patient, or if no response is obtained at the maximum tolerated dosage of TPM. It can be reduced in $100-200 \mathrm{mg} / \mathrm{day}$ decrements at weekly intervals without major problems.

\section{Patient counseling}

Patient counseling is important for creating appropriate expectations for both safety and efficacy. This should include a discussion of clinical goals with TPM therapy and establishment

Table: Topiramate dosage recommendations (add-on therapy in patients with refractory partial seizures).

\section{Recommended titration schedule}

- $25 \mathrm{mg} /$ day (h.s.) as starting dose

- Titrate in $25 \mathrm{mg} /$ day two-weekly increments to a dosage of 200 $\mathrm{mg} /$ day (100 mg bid)

- After the $200 \mathrm{mg} /$ day level, increase weekly by $50 \mathrm{mg} /$ day until clinical efficacy is achieved or the maximum tolerated dosage is reached.

\section{Target dosage}

- The minimum effective dosage of TPM is generally considered to be $200 \mathrm{mg} / \mathrm{day}$

- The effective dosage for most patients is $200-400 \mathrm{mg} /$ day.

Dosages $<200 \mathrm{mg} /$ day or up to $1600 \mathrm{mg} /$ day may be useful in some patients. 
of a reasonable timeline for assessing outcome. For many patients, an effective dosage will not be reached until the eighth week. The patient should also be informed concerning the side effects commonly experienced with TPM during the titration period and the time period during which resolution usually occurs.

\section{Conclusion}

TPM is a novel AED with established efficacy in partial and generalised epilepsies. ${ }^{11}$ Most side effects encountered with TPM in the add-on situation occur early in treatment therapy and tend to resolve with continuing therapy. Initial titration can be accomplished in $25 \mathrm{mg} /$ day increments at two-weekly intervals and the target dosage for most patients receiving TPM as adjunctive therapy is $200-400 \mathrm{mg} /$ day. When the optimal dosage of TPM has been reached, reduction or withdrawal of other AEDs can be considered.

\section{REFERENCES}

1. Walker MC, Sander JWAS. Difficulties in extrapolating from clinical trial data to clinical practice: the case of antiepileptic drugs. Neurology 1997; 49: 333-337.
2. Walker MC, Sander JWAS. The impact of new antiepileptic drugs on the prognosis of epilepsy: seizure freedom should be the ultimate goal. Neurology 1996; 46: 912-914.

3. Faught E, Wilder BJ, Ramsay RE, et al. Topiramate placebo-controlled dose-ranging trial in refractory partial epilepsy using 200-, 400-, and 600-mg daily dosages. Neurology 1996; 46: 16841690.

4. Privitera M, Fincham R, Penry J, et al. Topiramate placebo-controlled dose-ranging trial in refractory partial epilepsy using 600 $800-$, and 1,000-mg daily dosages. Neurology 1996; 46: 16781683 .

5. Ben-Menachem E, Henriksen O, Dam M, et al. Double-blind, placebo-controlled trial of topiramate as add-on therapy in patients with refractory partial seizures. Epilepsia 1996; 37: 539543.

6. Sachdeo R, Reife R, Lim P, Pledger G. Single-center topiramate monotherapy trial. Epilepsia 1995; 36 (Suppl 3): 151.

7. Biton V. Preliminary open-label experience with topiramate in primary generalized seizures. Epilepsia 1997; 38(Suppl 1): S42-\$45.

8. Espe-Lillo J, Ritter FJ, Frost MD, Spiegel RH, Reife R. Topiramate in childhood epilepsy: titration, adverse events, and efficacy in multiple seizure types. Epilepsia 1995; 36 (Suppl 4): 56.

9. Shorvon S. Safety of topiramate: adverse events and relationships to dosing. Epilepsia 1996; 37 (Suppl 2): 18-22.

10. Bourgeois BFD. Drug interaction profile of topiramate. Epilepsia 1996; 37 (Suppl 2): 14-17.

11. Walker MC, Sander JWAS. Topiramate: a new antiepileptic drug for refractory epilepsy. Seizure 1996; 5: 199-203. 